\title{
LINE-OF-SIGHT UNDERWATER WIRELESS COMMUNICATION SYSTEM
}

\author{
Suleman M. Ali Shaban ${ }^{1}$
}

Central organization of research and development, Laser guidance department.

Fathi Mohmed Omer Amer ${ }^{2}$

University of Gharyan, Physics Department.

Giuma Saleh Isa Abudagel ${ }^{3}$,

Azzaytuna University, Physics Department

DOI: 10.31364/SCIRJ/v8.i7.2020.P0720XX

http://dx.doi.org/10.31364/SCIRJ/v8.i7.2020.P0720XX

\section{ABSTRACT}

Underwater optical wireless communication (UOWC) has a number of potential applications ranging from deep oceans to coastal waters. However, the greatest challenge for UOWC originates from the fundamental characteristics of sea water or ocean; tackling these challenges requires a detailed understanding of complex physio-chemical biological systems. There are numerous similarities between UOWC and free space optical (FSO) communications, mainly because both are using optical wavelengths to transfer secure information between dedicated point-to - point connections. The aim of this paper is to provide detailed analysis of the line-of-sight LOS link, including the effect of the coefficients of absorption, scattering, and extinction on the received signal $\left(P_{r}\right)$ for three types of water, clean ocean water, coastal ocean water and turbid harbor water. A numerical underwater channel model developed in MATLAB software is provided for estimating the (Pr) under various link conditions. It illustrates the effect of different parameters such as the perpendicular distance between the transmitter and the receiver plane $(d)$, and the angle between the perpendicular to the receiver plane and the transmitter-receiver trajectory $(\theta)$ on the $\left(P_{r}\right)$. the provided results indicate that as the value of the $\theta$ increased $\left(P_{r}\right)$ decreased, and. $\left(P_{r}\right)$ also decreased by increasing $(d)$. It is obvious that the maximum range is provided by a line of sight using a narrow beam divergence; however, in this case, a precise positioning of the two platforms is required.

Keywords: Underwater optical wireless communication, Optical signal Attenuation due to the seawater, Ideal transmission wavelength of various types of water.

www.scirj.org

(C) 2020, Scientific Research Journal http://dx.doi.org/10.31364/SCIRJ/v8.i7.2020.P0720XX

This publication is licensed under Creative Commons Attribution CC BY. 


\section{INTRODUCTION}

Underwater optical wireless communications (UOWC) present a lot of similarities compared to free space optical communications mainly due to the fact that they employ optical wavelengths to transfer secure information between dedicated point-to-point links. The Basic Idea of (UOWC) system is to transmit information via light signals. For that reason, selected type of light is very important. Because various colors of light are absorbed in different levels underwater, every depth level has a different band characteristic. Blue light has the most efficient frequency to travel at deepest level underwater [1]. After $(15-20 \mathrm{~m})$ depth purple and blue colors, having the most refraction, cover much more space in the spectrum. Above $(3 \mathrm{~m})$ depth spectrum is very near today light. Blue-green wavelengths are can prove high bandwidth communications over moderate distance up to 100 meters.

Underwater optical link configurations have three basic types: (i) direct line-of- sight LOS links, (ii) NLOS links, and (iii) retro-reflector links. In this work, we analyze the performance of links based on line-of - sight connections. Configuration of point-to - point LOS is the most common link configuration used in UOWCs. The receiver is placed in this arrangement in such a way as to detect the light beam directly directed in the direction fixed by the transmitter. This system usually uses light sources with small angles of divergence, generally lasers, and requires precise transmitter-receiver pointing. This limitation will significantly limit the efficiency of UOWC systems in turbulent water environments, and can cause serious problems when transmitter - receiver are non-stationary nodes. The basic disadvantage of UOWC is that the water is a medium that extremely absorbs optical signals; the second problem is optical scattering due to all particles present in the sea. However, seawater shows a decreased absorption in the green / blue region of the visible light spectrum. Minimum attenuation is centered near $0.460 \mu \mathrm{m}$ in clear waters and shifts to higher values for dirty waters approaching $0.540 \mu \mathrm{m}$ for coastal waters [2, 3].

This paper is organized as follows: In section 3 the principal components of LOS communication link including optical transmitter, underwater wireless water channel, and optical Receiver are described in detail and this section provides the description of various challenges imposed by propagation of optical beam through underwater channel. In section 4 we describe the configuration scenario for direct LOS link. Finally, a numerical example to calculate the received power $\left(P_{r}\right)$ for clean ocean, coastal ocean and turbid harbor respectively at various perpendicular distance between the transmitter and the receiver plane $(d)$, and angle between the perpendicular to the receiver plane and the transmitter-receiver trajectory $(\theta)$ is presented in section 5 . 


\section{THE PRINCIPAL COMPONENTS OF LOS COMMUNICATION LINK}

A LOS communication link can be schematized in three parts,

1. Optical transmitter (LEDs or LDs).

2. Underwater wireless water channel.

3. Optical receiver module.

The input digital information stream modulates an optical source directly or via an external optical modulator. The common optical sources used in UWOC applications are laser diodes (LDs) and light emitting diodes (LEDs). Projection optics system is used to maximize and control the received optical signal, because optical beams diverge significantly with the propagation distance. The underwater wireless optical channel attenuates and distorts the optical signal. The receiver system usually consists of collection optics and a photodetector along with an appropriate electronic circuit. In UOWC system avalanche photodiode (APD) is the standard technology for optical receivers. Conceptual block diagram of a typical UWOC link is shown in figure (3.1).

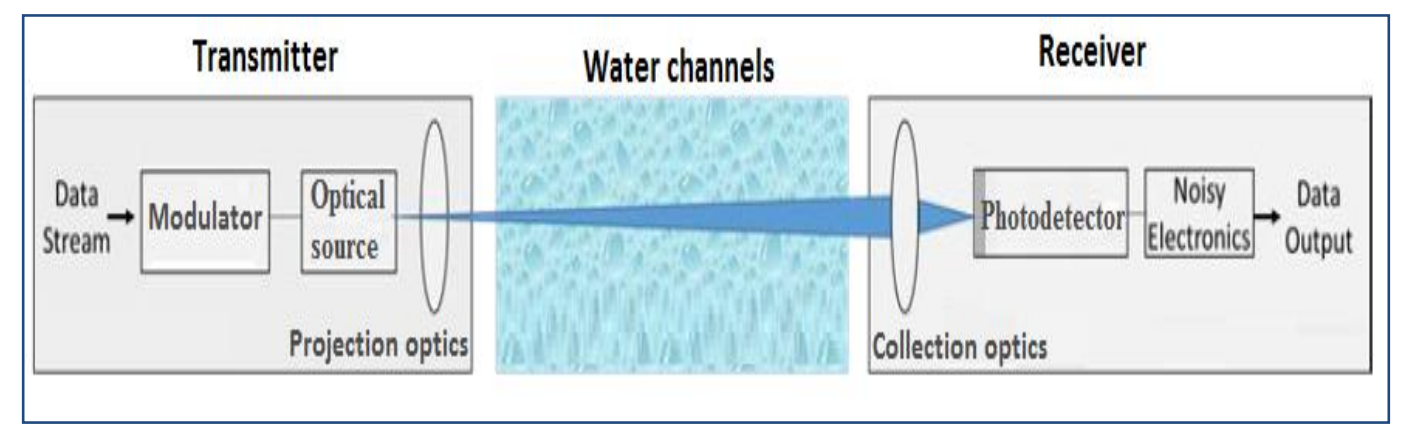

Figure (3.1). Conceptual block diagram of a typical UWOC link.

UWOC has high communication security, low implementation costs, low link delay, and high transmission data rate. UWOC can achieve a data rate on the order of (Gbps) over moderate distances of $(10 \mathrm{~m})$. The advantage of the high-speed will guarantee the realization of many real-time applications like underwater video transmission. UWOC systems are used for high speed underwater communications between mobile nodes or multiple fixed. They have great potential for applications as shown in figure (3.2). It shows several platforms (divers, submarines, ships, submarine sensors, etc.) connected by beams of light. 


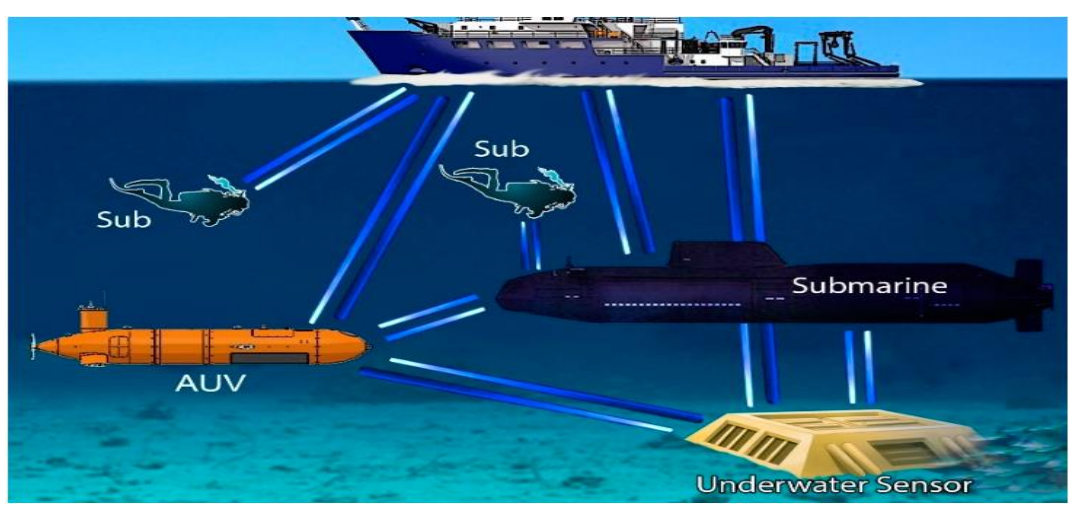

Figure (3.2) Typical application scenarios of UWOC.

\subsection{Optical Transmitter}

The optical transmitter's role is to transfer the data available in an electrical form into an optical form, and to launch the optical signal into a communication channel. (LDs) are generally used as optical light sources, (LEDs) can be used for some less-demanding applications. In both cases, the output of the source is in the form of an electromagnetic wave of constant amplitude. The modulator role is to impose the electrical data on this carrier wave by changing its phase, or amplitude, or both of them. In the case of some less-demanding applications, the current injected into a (LDs) itself is modulated directly, alleviating the need of an expensive modulator. Figure (3.3) shows the block diagram of an optical transmitter consisting of an optical source, a data modulator, and electronic circuitry used to derive them.

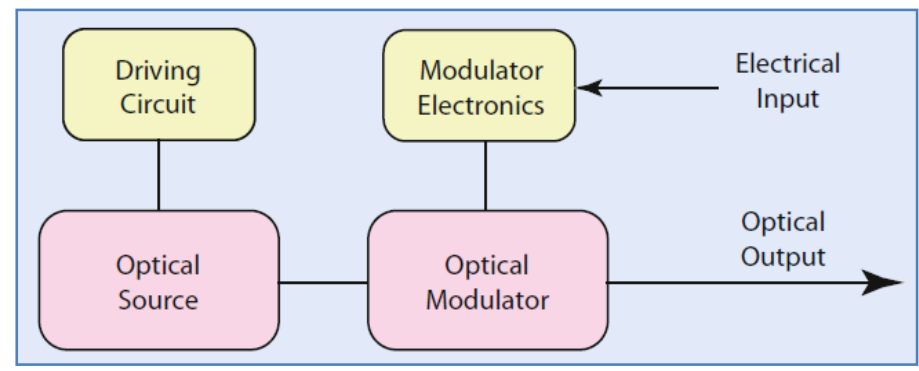

Figure (3.3) Components of an optical transmitter.

Selecting the photon light source drives the design of the rest of the optical transmitter. Though any light photon source, from an incandescent lamp to a laser, could be used, the size, power and switching speed constraints placed on the system narrowed down the selection to two feasible choices: (LEDs) and (LDs). LEDs have been operated from the near ultraviolet to the infrared region. Table (3.1) shows a comparison of various high-power LEDs. 


\section{Table (3.1) A comparison of high-powered LEDs on the market}

\begin{tabular}{|l|c|c|}
\hline \multicolumn{1}{|c|}{ Manufacturer and Model } & Wave Length (nm) & Luminous Flux (Im) \\
\hline Lamina Atlas NT-42C1-0484 & & 63 \\
\hline AOP LED Corp PU-5WAS & $460-470$ & 54 \\
\hline Kingbright AAD1-9090QB11ZC/3 & $455-475$ & 460 \\
\hline Ligitek LGLB-313E & 460 & 30.6 \\
\hline Toshiba TL12B01(T30) & $460-475$ & 6 \\
\hline Lumex SML-LX1610USBC & 460 & 5 \\
\hline High Powered Amber LED(26) & 470 & 130 \\
\hline Typical(not high-power) LED & 590 & $<1$ \\
\hline Luxeon Rebel LXML-PBO1-0023 & 470 & 48 \\
\hline
\end{tabular}

Laser diodes operating in the visible band are usually fabricated from $\mathrm{Ga}_{0.5} \mathrm{In}_{0.5} \mathrm{P}$ and generate light at $\lambda_{\mathrm{o}} \approx 0.670 \mu \mathrm{m}$. They use either gain-guided or index-guided structures. $\mathrm{CW}$ output powers are typically $(\approx 5 \mathrm{~mW}$ at $\mathrm{T}=300 \mathrm{~K})$; an off-the-shelf device might operate at a voltage of $(2.1 \mathrm{~V})$ and a current of $(85 \mathrm{~mA})$. Table (3.2) gives an overview of laser sources used in UWOC system.

\begin{tabular}{|c|c|c|c|}
\hline Type & Wavelength & Advantages & Disadvantages \\
\hline Argon-ion & $455-529 \mathrm{~nm}$ & High output & $\begin{array}{l}\text { Low efficiency; needs high } \\
\text { input power; needs cooling. }\end{array}$ \\
\hline $\mathrm{Nd}$ : YAG & $532 \mathrm{~nm}$ (green) $473 \mathrm{~nm}$ (blue) & $\begin{array}{l}\text { Very high output power; } \\
\text { long life time; compact. }\end{array}$ & $\begin{array}{l}\text { Variable efficiency; costly; } \\
\text { can be hard to modulate. }\end{array}$ \\
\hline Ti: Sapphire & $455 \mathrm{~nm}$ & Ultra fast output; tunable & Costly; sensitive to vibrations \\
\hline Metal vapour & $441.6 \mathrm{~nm}, 570 \mathrm{~nm}$ and $578 \mathrm{~nm}$ & $\begin{array}{l}\text { High output power; long } \\
\text { life time }\end{array}$ & Requires cooling \\
\hline Dye & 450nm-530nm & $\begin{array}{l}\text { Very high output power; } \\
\text { tunable; high date rate }\end{array}$ & $\begin{array}{c}\text { Costly; requires cooling } \\
\text { arrangements }\end{array}$ \\
\hline Semiconductor & $\begin{array}{l}405 \mathrm{~nm} \& 450-470 \mathrm{~nm} \\
(\mathrm{InGaN}) ; 375 \mathrm{~nm}-473 \mathrm{~nm}(\mathrm{GaN})\end{array}$ & High efficiency; compact & $\begin{array}{l}\text { Costly; easily damaged due } \\
\text { to over current }\end{array}$ \\
\hline
\end{tabular}

Both LDs and LEDs have weaknesses and strengths when it comes to underwater free space optical communications. LDs have higher optical power output and can switch faster, but www.scirj.org

(C) 2020, Scientific Research Journal http://dx.doi.org/10.31364/SCIRJ/v8.i7.2020.P0720XX

This publication is licensed under Creative Commons Attribution CC BY. 
LEDs are cheaper, simpler, and more confident. Though the coherence and minimal divergence of LDs is optimal for optical fiber communication systems, it does not play as big a role in wireless optical communication. Additionally, though LDs can switch faster, LEDs Can switch quick enough for our application ( $>=1 \mathrm{MHz}$ ). Since the goal is to have a cheap, small, reliable communication system that at least can handle (1 MHz), LEDs were chosen as the photon source for the optical transmitter. Table (3.3) shows the Comparison of LEDs and LDs.

\begin{tabular}{|c|c|c|}
\hline Characteristic & LED & Laser Diod \\
\hline Optical Spectral Width & $25-100 \mathrm{~nm}$ & 0.01 to $5 \mathrm{~nm}$ \\
\hline Modulation Bandwidth & $<200 \mathrm{MHz}$ & $>1 \mathrm{GHz}$ \\
\hline $\begin{array}{l}\text { Minimum Output Beam } \\
\text { Divergence }\end{array}$ & Wide (about $0.5^{\circ}$ ) & Narrow (about $0.01^{\circ}$ ) \\
\hline Temperature Dependency & Little & Very temperature dependent \\
\hline Special Circuitry Required & None & $\begin{array}{l}\text { Threshold and temperature compensation } \\
\text { circuitry }\end{array}$ \\
\hline Cost & $\begin{array}{l}\text { Low, off-the-shelf } \\
\text { components available }\end{array}$ & High, need specialized optics and electronics \\
\hline Lifetime & $\begin{array}{c}\text { Long, with little } \\
\text { degradation of power } \\
\text { levels }\end{array}$ & Medium, power levels degrade over time \\
\hline Reliability & High, $10^{8}$ hours & Moderate, $10^{5}$ hours \\
\hline Coherence & Incoherent & Coherent \\
\hline Eye Safety & Eye safe & Must take precautions \\
\hline
\end{tabular}

\subsection{Underwater Wireless Water Channel}

Compared with terrestrial free space optical communication FSO channels, UWOC channels have several unique characteristics. Existing terrestrial FSO channel models are not apposite for underwater environment; therefore, another reliable channel models must be proposed and studied. In order to derive new channel models for UWOC, we have to firstly understand the basic properties of light propagation in the underwater environment. According to Mobley's statements in [4]. The optical properties of water can be divided into two different groups:

I. Inherent Optical Properties (IOPs): describe those optical parameters that depend only on the transmission medium itself. They are independent of the light sources WwW.scirj.org 
characterizations. The major IOPs of water are the scattering coefficient, the absorption coefficient, the attenuation coefficient, and the volume scattering function.

II. Apparent Optical Properties (AOPs): are known as the optical parameters that are not dependent only by the medium, but also the geometrical structure of the light illumination including therefore directional properties. The three major AOPs of water are reflectance, radiance and irradiance.

In a UWOC system, IOPs are usually used in determining communication link budgets, while AOPs are used to calculate the level of ambient light for communication systems near the surface of the ocean.

Absorption and scattering coefficients are the two major IOPs that determine the underwater optical signal attenuation. In general, three undesirable effects can be caused by the impacts of absorption and scattering to a UWOC system.

1. When absorption is present, the total energy of light propagation is increasingly decreasing; which will limit the link distance of the UWOC.

2. When scattering is present, since the size of optical aperture is finite, scattering will spread the beam of light and result in a reduction of the photons number which collected by the receiver.

3. Due to the scattering of optical signal in an underwater environment, each photon may arrive at the receiver panel in different time slots, and multi-path dispersions will take place. Figure (3.4) illustrates the geometric model for inherit optical properties.

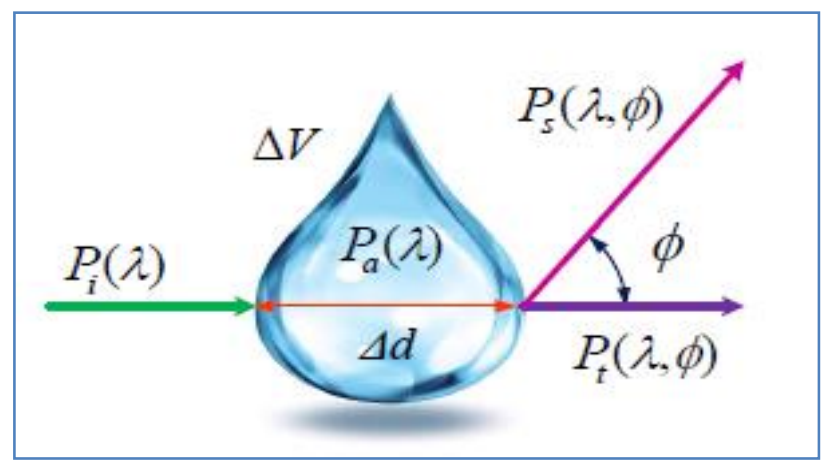

Figure (3.4) Geometric model for inherit optical properties [5].

We are supposing a volume of water $(\Delta V)$ with thickness $(\Delta d)$ is illuminated by a collimated beam of light with wavelength $(\lambda)$. And the power of the incident light is denoted as $\left(P_{i}\right)$. Water absorbs a portion of incident light power $\left(P_{a}\right)$, and another portion of light power $\left(P_{\mathrm{s}}\right)$ is scattered. $\left(P_{t}\right)$ is the remaining light power that will propagate as desired. 
The characteristics of different bodies of water vary with their geographical location (from deep Blue Ocean to near-land coastal waters) and the concentration of dissolved substances. The marine medium contains approximately 80 different components, dissolved or suspended in pure water, with varying concentrations. Some of them are given below [6,7].

- Various dissolved salts, such as $\mathrm{NaCl}, \mathrm{MgCl} 2$, etc., which absorb light at specific wavelengths and cause dispersing effects.

- Detrial and mineral elements, such as sand, metal oxides, contributing to both scattering and absorption.

- Colored organic dissolved matter such as fulvic and humic acids that influence absorption, mostly in blue and ultraviolet wavelengths.

- Organic matters such as viruses, bacteria and organic detritus that add backscattering, especially in the blue spectrum

- Chlorophyll-A phytoplankton that absorbs extremely in the blue-red zone and disperses green light.

- Pytoplankton with chlorophyll-A, which disperses green and strongly absorbs light in the blue-red zone.

Since chlorophyll absorbs the red and blue wavelengths and the particles contribute significantly to the scattering coefficient, its concentration $\mathrm{C}\left(\mathrm{in} \mathrm{mg} / \mathrm{m}^{3}\right)$ can be used as the free parameter to measure the absorption and dispersion coefficients [8,9].Figure (3.5) shows the absorption and scattering coefficients of water with $1 \mathrm{mg} / \mathrm{m}^{3}$ of chlorophyll concentration.

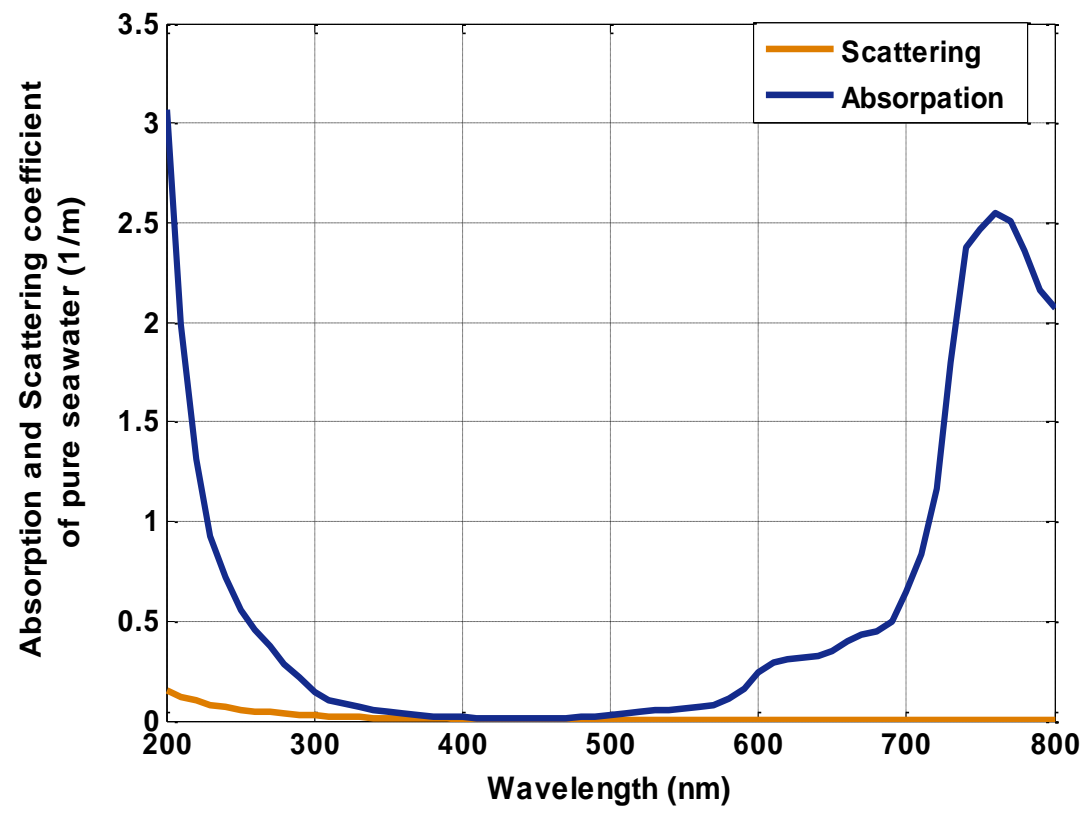

Figure (3.5) Absorption and scattering coefficients of water with $1 \mathrm{mg} / \mathrm{m}^{3}$ of chlorophyll concentration. 
The four main types of water commonly listed in the literature are as follows:

1. Pure sea water: Absorption in pure sea water is considered as the sum of absorption in pure water and the absorption of salt in pure salt water. It is assumed to be negligible in visible light spectrum $(0.4-0.7 \mu \mathrm{m})$. Absorption here is that the main limiting factor that increases with increases in wavelength. Therefore, the red wavelength of $(0.5 \mu \mathrm{m})$ is more attenuated than the blue light, which is why deep clear ocean water is rich in blue colour. In pure seawater the water absorption coefficient is given as [10].

$$
\alpha_{\text {sea water }}(\lambda)<K(\lambda)-\frac{b(\lambda)}{2}
$$

Where:

$\lambda$ : is the operating wavelength,

$K$ :is the diffuse coefficient and

$b:$ is the scattering coefficient.

In this case, the propagation of the optical beam is mostly in straight line due to the low value of $\mathrm{b}$ and $\mathrm{K}$.

2. Ocean water: contains higher concentrations of dissolved particles such as dissolved salts, colored dissolved organic matter, mineral components etc. Depending on the concentration of suspended particles and their geographical location, they are further categorized into Type 1 - 3 (based on Jerlov water type) [11].

3. Coastal ocean water: it has a higher concentration of dissolved particles and thus increases the turbidity level. In this type of water, the effect of scattering and absorption is more.

4. Turbid harbor: it has the largest concentration of dissolved and suspended particles and thus, limits the propagation of optical beam due to absorption and scattering.

Based on the attenuation coefficient that has been introduced, Beer-Lambert law offers the easiest and most commonly used scenario to explain the light attenuation effects in underwater environment as follows:

$$
P(z)=P_{o} e^{-c(\lambda) z}
$$

Where:

$P_{o}:$ is the power of transmitted light,

$\mathrm{Z}$ : denotes the light transmission distance,

$P$ : represents the power of light after transmitting $\mathrm{z}$ distance, and

$c(\lambda)$ : is the extinction coefficient expressing the total attenuation occurred by the propagation through the water.

The total attenuation can be described as the sum of absorption and scattering. In the case of a fully absorbing or completely dispersing medium, the total attenuation coefficient in Equation (3.2) may be replaced by the absorption coefficient (a), or scattering coefficient (b).

www.scirj.org

(C) 2020, Scientific Research Journal http://dx.doi.org/10.31364/SCIRJ/v8.i7.2020.P0720XX

This publication is licensed under Creative Commons Attribution CC BY. 
The term (cz) is also referred as attenuation length, contributes on the decreasing of the received power by a factor of $\exp (-1)$, or $\sim 63 \%$ [12]. Based on this, we got:

$$
c(\lambda)=\alpha(\lambda)+b(\lambda)
$$

The exact attenuation coefficient value $\mathrm{c}(\lambda)$ can vary with various types of water and water depth. The typical values associated with four major types of water are given in Table (3.4) for $\mathrm{a}(\lambda), \mathrm{b}(\lambda)$, and $\mathrm{c}(\lambda)$.

\section{Table (3.4) Typical values of $a(\lambda), b(\lambda)$, and $c(\lambda)$ for different water types.}

\begin{tabular}{|c|c|c|c|}
\hline Water types & $a(\lambda)\left(\mathrm{m}^{-1}\right)$ & $b(\lambda)\left(\mathrm{m}^{-1}\right)$ & $c(\lambda)\left(\mathrm{m}^{-1}\right)$ \\
\hline Pure sea water & 0.053 & 0.003 & 0.056 \\
\hline Clear ocean water & 0.114 & 0.037 & 0.151 \\
\hline Costal ocean water & 0.179 & 0.219 & 0.298 \\
\hline Turbid harbor water & 0.295 & 1.875 & 2.17 \\
\hline
\end{tabular}

The absorption coefficient $a(\lambda)$ and the scattering coefficient $b(\lambda)$ can thus be represented as a function of the wavelength $\lambda$ and the concentration $C[13]$ :

$$
\begin{gathered}
\mathrm{a}(\lambda)=\left[a_{w}(\lambda)+0.06 a_{c}(\lambda) C^{0.65}\right]\{1+0.2 \exp [-0.014(\lambda-440)]\} \\
\mathrm{b}(\lambda)=0.30\left(\frac{550}{\lambda}\right) C^{0.62}
\end{gathered}
$$

where $a_{w}$ points out the coefficient of pure water absorption while, $a_{c}$ is a non-dimensional number, statistically derived that points out the absorption coefficient specific for the chlorophyll. Thus, The Concentration of Chlorophyll C, expressed in $\left(\mathrm{mg} \cdot \mathrm{m}^{3}\right.$ ), may be used as the free parameter for calculating $a(\lambda)$ and $b(\lambda)$. Figure (3.6) depicts the absorption, scattering, and extinction coefficients for four types of water, pure sea water, clean ocean water, coastal ocean water, and turbid harbor water. It is important to note that the absorption measurements have been obtained in a spectral band with $\lambda$ centered at $532 \mathrm{~nm}$. 


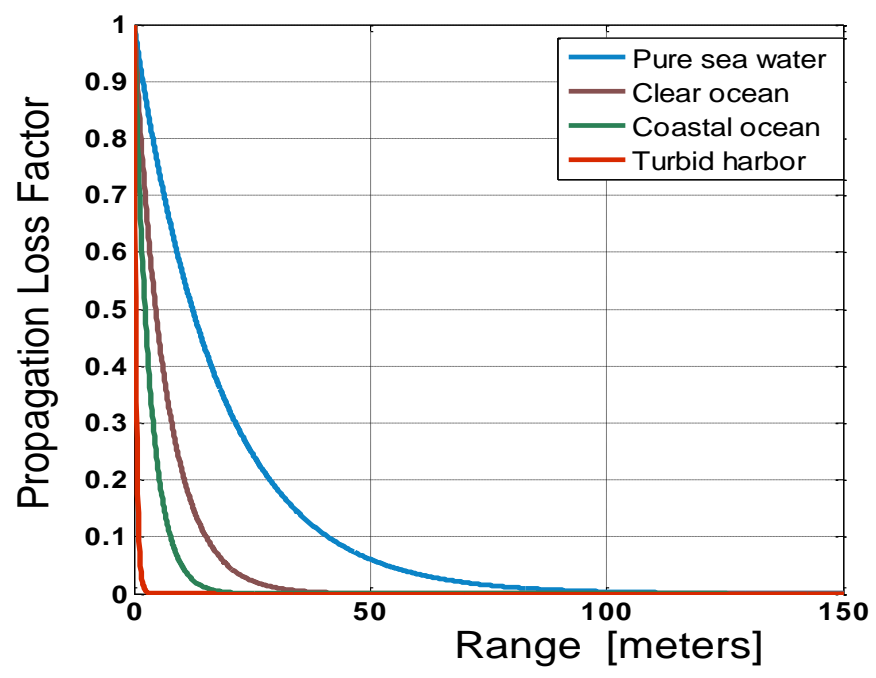

Figure (3.6) absorption, scattering, and extinction coefficients for four types of water,

The minimum attenuation window for different types of water will be different, as different factors affect the absorption in underwater environments. Table (3.5) lists ideal wavelengths for transmission of different types of water.

\begin{tabular}{|l|c|c|c|}
\hline \multicolumn{4}{|c|}{ Table (3.5) Ideal transmission wavelength of various types of water. } \\
\hline \multicolumn{1}{|c|}{ Water Type } & $\begin{array}{c}\text { Chlorophyll } \\
\text { concentration }\end{array}$ & $\begin{array}{c}\text { Humic and Fulvic } \\
\text { concentration }\end{array}$ & Operation Wavelength \\
\hline Pure sea/clear ocean & Less & Less & Blue-green $(450-500 \mathrm{~nm})$ \\
\hline Coastal ocean & High & High & Yellow-green $(520-570 \mathrm{~nm})$ \\
\hline Turbid harbor & Very High & Very High & Yellow-green $(520-570 \mathrm{~nm})$ \\
\hline
\end{tabular}

\subsection{Optical Receiver}

An LOS communication system requires a receiver (photodetector) to detect the optical signal and transfer it into an electrical signal. The photodetector component in the LOS communication sensors plays an important role in determining the system-level parameters including sensitivity, spectral operating band, and resolution. The spectral response is determined by the photodetector material characteristics and the operating temperature. The photodetector sensitivity is a function of material (i.e., band gap), bandwidth, wavelength, detector size. The block diagram of an optical receiver in LOS communication system is shown in figure (3.7). It is composed of a photodetector and a demodulator along with the electronic circuitry used to derive them. Semiconductor photodiodes are used as photodetector because of their compact size and low cost. 


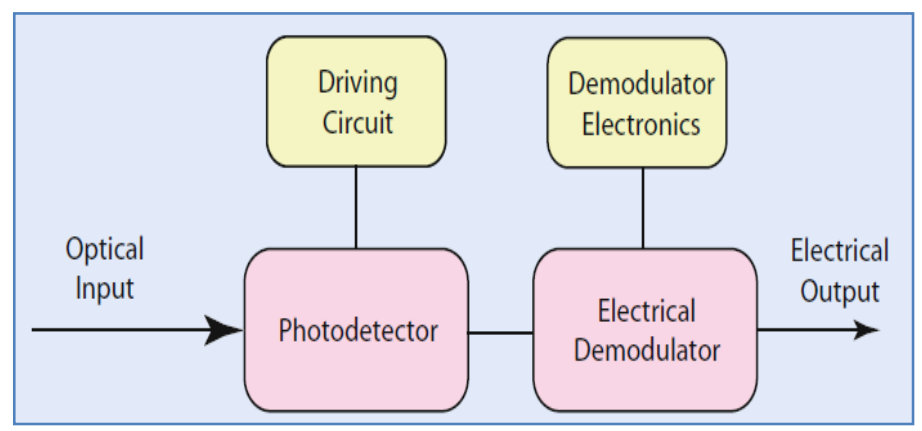

Figure (3.7) block diagram of an optical receiver.

There are two types of photodetectors that are of primary interest, i.e. Positive-IntrinsicNegative (PIN) and Avalanche photodiode (APD). The characteristics of these types of photodiodes have been discussed by M. Azadeh [14]. Table (3.6) shows comparison of PIN and APD. These PIN data provide higher bandwidth, higher sensitivity, lower operating voltage and cheaper as well.

\begin{tabular}{|c|c|c|}
\hline Variable & PIN & APD \\
\hline Materials & \multicolumn{2}{|c|}{ Si, Ge, InGaAs } \\
\hline Bandwidth & \multicolumn{2}{|c|}{ To $40 \mathrm{GHz}$} \\
\hline Life time & \multicolumn{2}{|c|}{ OK } \\
\hline Spectral range & \multicolumn{2}{|c|}{ Tunable (Ultraviolet, Visible light, Near Infra-red) } \\
\hline Form factor & \multicolumn{2}{|c|}{ Small } \\
\hline Electromagnetic immunity & \multicolumn{2}{|r|}{ No } \\
\hline Magnetic field sensitivity & \multicolumn{2}{|r|}{ No } \\
\hline Large area & \multicolumn{2}{|r|}{ No } \\
\hline Gain & 1 & $10^{2}$ \\
\hline Operating Voltage (V) & $\operatorname{Low}(0-5)$ & High(100 -100k) \\
\hline Cost & Low & High \\
\hline Efficiency (A/W) & Low & High \\
\hline Response time & Fast & Slow \\
\hline Sensing sensitivity & Low & High \\
\hline Temperature sensitivity & Low & High \\
\hline Bandwidth \& bit rate & High & Medium \\
\hline Damage by Stray light & No & Yes \\
\hline Dark current & High & Low \\
\hline Excess noise factor & Low & Medium \\
\hline Mechanical Robustness & High & Medium \\
\hline
\end{tabular}


The demodulator design is based on the modulation scheme used at the transmitter. Many optical communications systems use a binary scheme called direct-detection intensity modulation. In this case, demodulation is done by a decision circuit which identifies incoming bits as 1 or 0 , depending on the amplitude of the electrical signal. Owing to loss of any optical signal during its transmission and detection, all optical receivers make some errors, shot noise being the most fundamental cause of noise. A digital light wave system's performance is characterized by its bit-error rate. It is customary to define it as the average probability of identifying a bit incorrectly. Sometimes, the error-correction codes are used to improve an optical communication system's raw bit error.

\section{LINE-OF-SIGHT COMMUNICATION LINK SCENARIO}

Direct LOS link is the simplest, unobstructed and point-to-point underwater connection of transmitter to receiver. This link is quite well to implement in case of static transmitters and receivers such as two sensor nodes at the seabed of the ocean. Direct LOS configuration is used when distance is needed in areas where the concentration of chlorophyll and related constituents is small. Figure (4.1) shows the scenario of LOS link configuration, in this scenario, the transmitter directs the light beam toward the receiver. The optical signal which reaches the receiver is obtained by multiplying the transmitter power, gain of the telescope, and losses and is given by Ref. [15] as:

$$
P_{R-l o s}=P_{T} \eta_{T} \eta_{R} \exp \left[(\lambda), \frac{d}{\cos (\theta)}\right] \frac{A_{R} \cos \theta}{2 \pi d^{2}\left(1-\cos \theta_{d}\right)}
$$

where:

$P_{T}:$ the average transmitter optical power,

$\eta_{T}$ : the optical efficiency of the transmitter,

$\eta_{R}$ : the optical efficiency of the receiver,

$d$ : the perpendicular distance between the transmitter and the receiver plane,

$\theta$ : the angle between the perpendicular to the receiver plane and the transmitter-receiver trajectory,

$A_{R}$ : the receiver aperture area, and

$\theta_{d:}$ the laser beam divergence angle. Typically, the value of $\theta_{d}$ is $\left(\theta_{d}<<\pi / 20\right)$ [16].

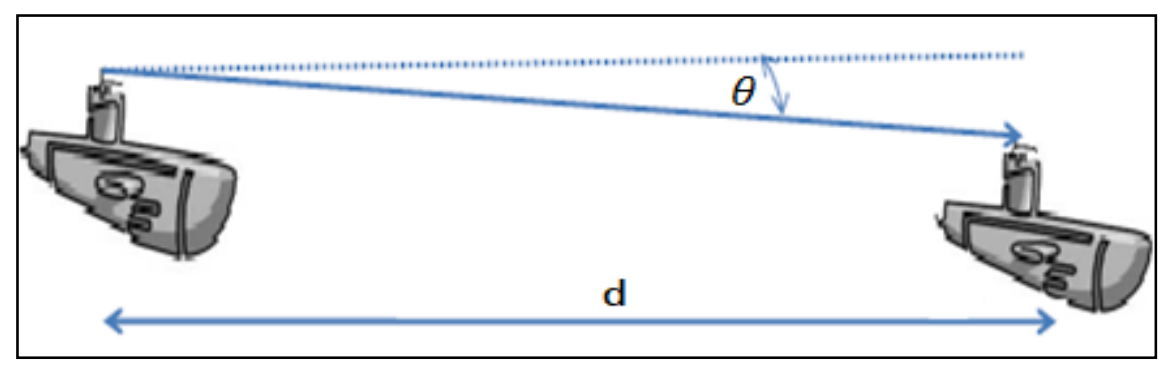

Figure (4.1) scenario of LOS link configuration.

WWW.scirj.org

(C) 2020, Scientific Research Journal http://dx.doi.org/10.31364/SCIRJ/v8.i7.2020.P0720XX

This publication is licensed under Creative Commons Attribution CC BY. 
To keep both transmitter and receiver bore-sighted, very sophisticated pointing and tracking mechanism is required for mobile platforms like AUVs. It works well in clear oceans where a narrow beam signal is directed by the transmitter towards the receiver. However, due to the growth of marine life, fish schools or other obstacles, there are great chances of obscuration. Therefore, it is important to design a system that discourages marine life from blocking the propagation path to establish a LOS link. The lights which are considered optimal for underwater optical communication also attract fish schools. Fishes in the ocean water prefer blue-green wavelengths while freshwater fish prefer yellow-green wavelengths. Thus, flashing or erratic lights are preferred to avoid fishes entering the LOS region [17].

\section{SIMULATIONS, RESULTS AND DISCUSSION}

The received power $\left(P_{r}\right)$ is calculated from the mathematical model expressed in equation (4.1) using the parameters illustrated in table (5.1). The received power is plotted against both the perpendicular distance between the transmitter and the receiver plane $(d)$, and the angle between the perpendicular to the receiver plane and the transmitter-receiver trajectory $(\theta)$ for clean ocean, coastal ocean and turbid harbor respectively, where ( $0 \leq d \leq 100$ meter), and $\left(0^{\circ} \leq \theta \leq 60^{\circ}\right)$.

\section{Table (5.1) Parameters Used For Numerical Calculation}

\begin{tabular}{|l|c|}
\hline \multicolumn{1}{|c|}{ Parameter } & Value \\
\hline Transmission wavelength $(\mathrm{nm})$ & 532 \\
\hline Extinction coefficient for Clean ocean & 0.151 \\
\hline Extinction coefficient for Coastal ocean & 0.298 \\
\hline Extinction coefficient for Turbid harbor & 2.17 \\
\hline Critical angle(degree) & 48.44 \\
\hline Optical efficiency for Retro reflector & 0.9 \\
\hline Optical efficiency for Transmitter & 0.9 \\
\hline Optical efficiency for Receiver & 0.9 \\
\hline Transmitter power(W) & 1 \\
\hline Receiver aperture area $\left(\mathrm{m}^{2}\right)$ & 0.01 \\
\hline Retro reflector aperture area $\left(\mathrm{m}^{2}\right)$ & 0.01 \\
\hline Retro reflector beam divergence $\left(\theta_{\text {retro }}\right)(\mathrm{deg})$ & 10 \\
\hline Beam divergence angle $\theta_{0}($ deg) & 68 \\
\hline Transmitter inclination angles $\theta_{\min }, \theta_{\max }$ & 0,68 \\
\hline Transmitter depth $\mathrm{h}(\mathrm{m})$ & 20 \\
\hline Receiver depth $x(m)$ & 20 \\
\hline
\end{tabular}




\subsection{Calculation of clear ocean water and costal ocean water}

The received power versus distance (10 -100 meter) is plotted for underwater direct LOS communication link for clean and coastal ocean water conditions as illustrating in figure (5.1). We noted that due to the least reduction of solar energy in the vertical direction the maximum power was for clean ocean.

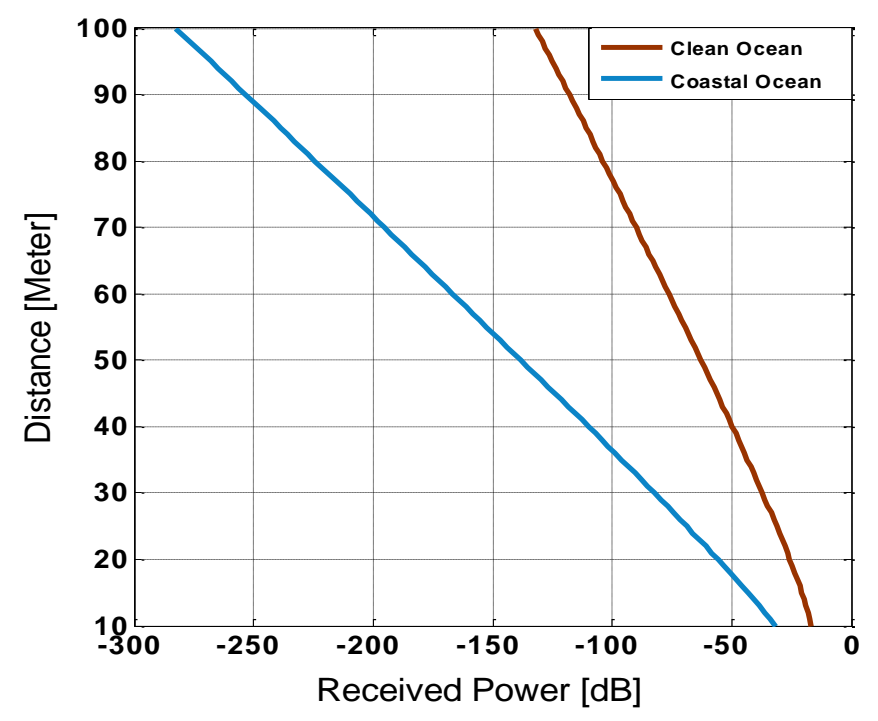

Figure (5.1) Scenario of LOS for clean and coastal ocean water conditions (variable distance)

The same procedure was repeated again for the power received versus angle ( 0 - 60 degree). The results are shown in the figure (5.2). It can be seen that the received power was found to decrease as the $\theta$ value increased. Received power also decreased with increasing the perpendicular distance between the transmitter and the receiver.

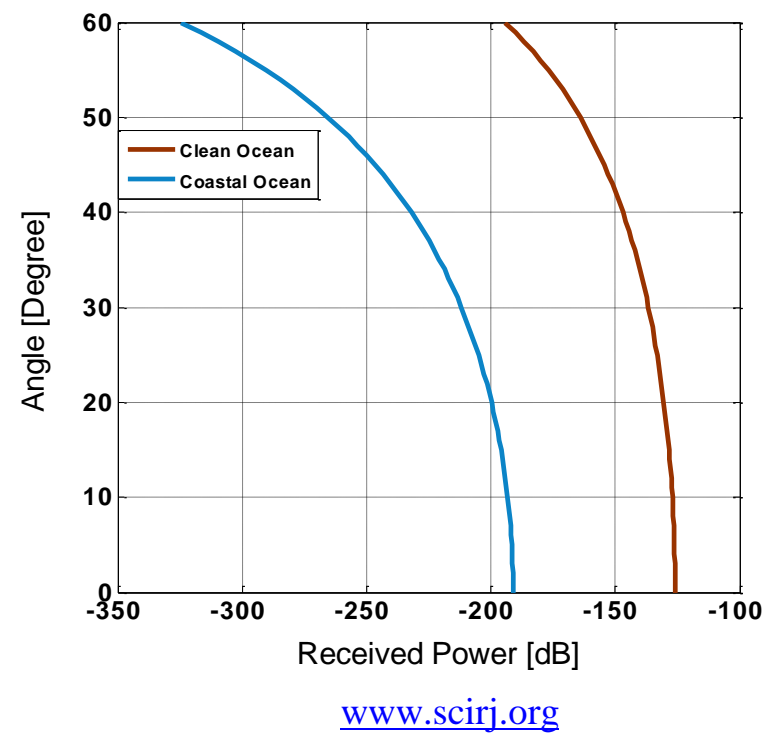

(C) 2020, Scientific Research Journal http://dx.doi.org/10.31364/SCIRJ/v8.i7.2020.P0720XX

This publication is licensed under Creative Commons Attribution CC BY. 
Figure (5.2) Scenario of LOS for clean and coastal ocean water conditions (variable angle)

\subsection{Calculation turbid harbor ocean water}

Finally, the procedure was repeated for the received power versus distance (10 - 100 meter) for underwater direct LOS communication link for turbid harbor water condition as shown in figure (5.3). It can be seen that the received power value was the least for turbid ocean.

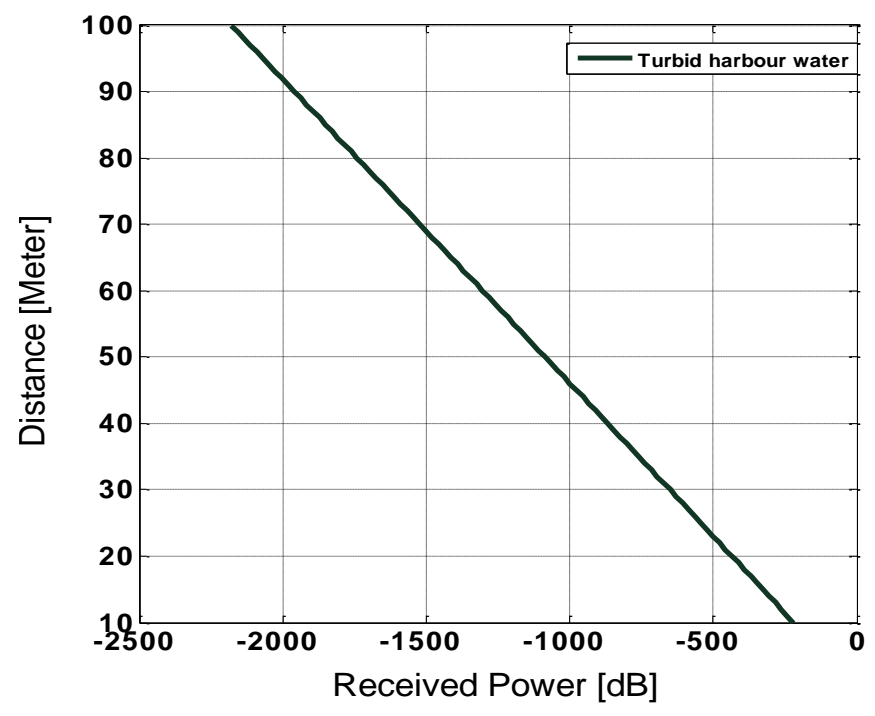

Figure (5.3) Scenario of LOS for turbid harbour water condition (variable distance)

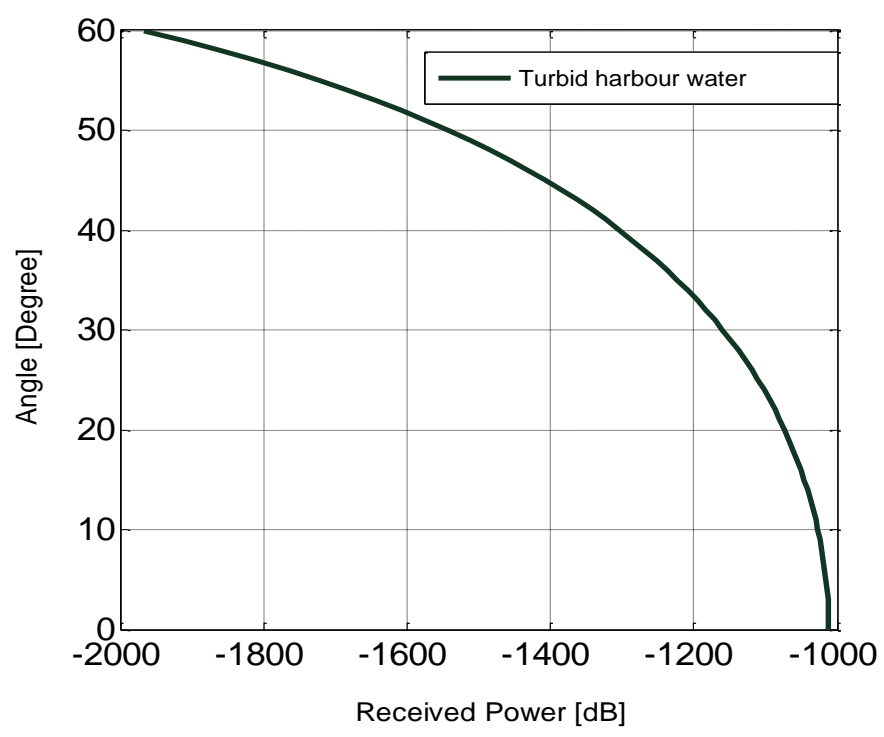

Figure (5.4) Scenario of LOS for turbid harbour water condition (variable angle)

www.scirj.org

(C) 2020, Scientific Research Journal http://dx.doi.org/10.31364/SCIRJ/v8.i7.2020.P0720XX

This publication is licensed under Creative Commons Attribution CC BY. 


\section{SUMMARY AND CONCLUSIONS}

A comparative analysis of underwater direct LOS scenario for three different parts of the ocean namely clean ocean, coastal ocean and turbid harbor was done, and the received power for these types of water was comparable.

In pure seawater, the main loss is due to absorption, although there is also a scattering loss in clear ocean water due to a higher concentration of particles compared to pure seawater. Coastal ocean water has even higher particle concentrations which affect scattering and absorption, while turbid harbor and estuary water have the highest particle concentration.

Results indicated that, because the turbid harbor had the highest extinction coefficient of (2.17), the power value obtained was the least for turbid ocean. Maximum power of this scenario was noted for clean ocean due to the least reduction of extinction coefficient. As the value of the $\theta$ increased, the power received decreased. Received power also decreased by increasing the perpendicular distance between the transmitter and the receiver.

It is obvious that the maximum range is provided by line of sight using narrow beam divergence; however, the precise positioning of the two platforms is required in this case. On the other hand, when it is necessary to simultaneously broadcast (for example) from a submarine to multiple platforms e.g. unmanned underwater vehicles (UUVs) or divers, the best choice is to use LOS with wide divergence of beams.

\section{References}

[1] Bruno, T.J. and Svoronos, P.D.N., CRC Handbook of Fundamental Spectroscopic Correlation Charts, CRC Taylor\&Francis Press, 2006.

[2] Young E.Y. S, Bullock A.M., (2003), "Underwater airborne laser communication system characterization of the cannel" Free-Space Laser Communication Technologies XV, G. Stephen Mecherle, Editor, Proceedings of SPIE, Vol. 4975

[3] Sathyendrenath S., (1984) "Inherent optical properties of natural seawater", Defence Science Journal, Vol. 34, No. 1, pp. 34, 1-18

[4] C. D. Mobley, Light and Water: Radiative Transfer in Natural Waters. San Diego, CA: Academic press, 1994.! pages vii, 12, 13, 14, 15, 18, 19, 23

[5] C. Mobley, Light and Water: Radiative Transfer in Natural Waters. Academic Press, 1994.

[6] Gupta S.K., (1984) "Spectral transmission studies of ocean water under different sea conditions", Defence Science Journal, Vol. 34, No. 1, pp. 19-28

[7] Gabriel C., Khalighi M.A., Bourennane S., Pierre Leon, Vincent Rigaud, (2013) "Montecarlo-based channel characterization for underwater optical communication systems" Journal of Optical Communications and Networking January 2013, Vol. 5, Issue 1, pp. 1-12 The Optical Society

www.scirj.org

(C) 2020, Scientific Research Journal http://dx.doi.org/10.31364/SCIRJ/v8.i7.2020.P0720XX

This publication is licensed under Creative Commons Attribution CC BY. 
[8] Haltrin V., (1999) "Chlorophyll-based model of seawater optical properties", Applied Optics, Vol. 38, No. 33, pp. 6826-6832

[9] Mobley C.D., (1994), "Light and Water: Radiative Transfer in Natural Waters", Book, Academic Press Inc. A division of Harcourt Brace and Company 525B Street Suite 1900, San Diego, California 92101-4495 United Kingdom Edition published by: Academic Press Limited, 24-28 Oval Road, London NW1 7DX

[10] R. C. Smith and K. S. Baker, “ Optical properties of the clearest natural waters (200_800 nm)," Appl. Opt., vol. 20, no. 2, pp. 177_184, 1981.

[11] J. R. Apel, Principles of Ocean Physics (International Geophysics Series), vol. 38. London, U.K.: Academic Press, 1987, pp. 509_584.

[12] Cochenour B., and Mullen L., (2012) "Free-space optical communications underwater", in Advanced Optical Wireless Communication Systems, (ed). S. Arnon, J.R. Barry, G.K. Karagiannidis, R. Schober, and M. Uysal, Advanced Optical Wireless Communication Systems Pages 273-302 Cambridge, UK: Cambridge Univ. Press

[13] Xu, J.; Song, Y.; Yu, X.; Lin, A.; Kong, M.; Han, J.; Deng, N. Underwater wireless transmission of high-speed QAM-OFDM signals using a compact red-light laser. Opt. Express 2016, 24, 8097-8109.

[14] M. Azadeh, PIN and APD detectors. Fiber Optics Engineering-Optical Networks, Springer Science+Business Media, 2009.

[15] S. Arnon and D. Kedar, "Non-line-of-sight underwater optical wireless communication network," J. Opt. Soc. Am. A 26(3), 530-539 (2009).

[16] S. Arnon, “Underwater optical wireless communication network," J. Opt. Eng., vol. 49, no. 1, pp. 015001-1_015001-6, 2010.

[17] C. Rich and T. Longcore, Eds., Ecological Consequences of Artificial Night Lighting. Washington, DC, USA: Island Press, 2006

\section{Authors:}

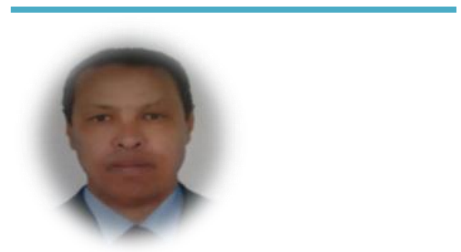

Suleman M. Ali Shaban

was born in Tripoli, Libya, in 1963. He received the BSc in physics from University of Tripoli Libya in 1988, M.S. degree in Laser Technology from University of Belgrade, Serbia, in 2003, He is a member at Central organization of research and development. Laser guidance department, Laser warring receiver unit [LWRS]. He is currently PhD Student at University of Singidunum, Electrical Engineering Dept / Belgrade Serbia.

Under the supervision of Dr Marko Tanasukovic

www.scirj.org

(C) 2020, Scientific Research Journal http://dx.doi.org/10.31364/SCIRJ/v8.i7.2020.P0720XX

This publication is licensed under Creative Commons Attribution CC BY. 


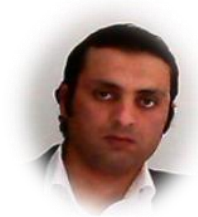

Fathi Mohmed Omer Amer

Was born in Libya, Komata in 1977. He received B Sc in physics from university of Al-jabal Al-gharby, Libya in 1998, MSc in physics (optoelectronics) from Belgrade university, He is currently lecturer in University of Gharyan, Faculty of Arts and Science, Physics Department.

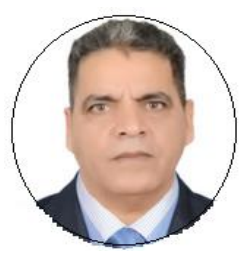

Giuma Saleh Isa Abudagel

was born in Tarhuna, Libya, in 1963. He received B Sc in physics from university of Tripoli, Libya in 1987, M.Sc. degree in Optoelectronics and Laser Technics from University of Belgrade, Serbia, in 2000, and the Ph.D. degree in Electrical Engineering, Nanoelectronics and Photonics from University of Belgrade, Serbia, in 2019, he is currently assistant Professor with Azzaytuna university, Faculty of Science, Physics Department, Libya. 